\title{
EVALUACIÓN DE LA ORGANIZACIÓN ESPACIAL DE UN AULA DESDE LA PERSPECTIVA DE UN GRUPO DE ESTUDIANTES UNIVERSITARIOS/AS: UN ESTUDIO DE CASO
}

Evaluation of the spatial organization of a classroom from the perspective of a group of university students: a case study

Avaliação da organização espacial de uma sala de aula na perspectiva de um grupo de estudantes universitários: um estudo de caso

\author{
Katia Álvarez Díaz (1)
}

José Ramón Márquez Díaz (2)

Freddy Franco-Morales (3)

(1) Universidad de Huelva, España. Teléfono: +34 675107359. Correo electrónico: katia.alvarez@alu.uhu.es

(2) Universidad de Huelva, España. Teléfono: +34 658672452. Correo electrónico: joseramon.marquez@alu.uhu.es

(3) Université de Sherbrooke, Canadá. Teléfono: +1 8199192943. Correo electrónico: freddy.franco.morales@usherbrooke.ca

\section{Resumen}

Este estudio es reflejo de la acuciante necesidad de la calidad e innovación de la práctica educativa en la formación del alumnado, donde la variable organizativa de toda acción pedagógica se considera un proceso complejo que sostiene la consideración de numerosos elementos: recursos, momentos y espacios en los que se desarrollan los procesos de enseñanza aprendizaje. El objetivo de la investigación es evaluar la organización espacial de un aula desde la perspectiva de un grupo de estudiantes universitarios/as. Para ello, durante el curso académico 2017/2018, a través de una metodología de corte cualitativo, se examina el caso de un aula universitaria. Se toma una muestra de 35 estudiantes. Para ello, se emplean diversas técnicas de investigación: entrevistas semiestructuradas y observación participante. Los resultados indican que, en general, la organización espacial del centro y, en particular, del aula ejerce un papel activo en todo proceso de enseñanza aprendizaje. En este sentido, la didáctica y la organización espacial deben unificarse para el correcto desarrollo integral del alumnado.

Palabras clave: Educación superior; organización espacial; aula; evaluación

Evaluación de la organización espacial de un aula desde la perspectiva de un grupo de estudiantes universitarios/as: un estudio de caso 


\begin{abstract}
This study reflects the pressing need for quality and innovation of the educational practice in the training of students, where the organizational variable of all pedagogical action is considered a complex process that supports the consideration of numerous elements: resources, moments and spaces in those that develop the teaching-learning processes. The aim of the research is to evaluate the spatial organization of a classroom from the perspective of a group of university students. For this, during the academic year 2017/2018, through a methodology of qualitative cut, the case of a university classroom is examined. A sample of 35 students is taken. For this, several research techniques are used: semi-structured interviews and participant observation. The results indicate that, in general, the spatial organization of the center and, in particular, of the classroom, plays an active role in every teaching-learning process. In this sense, the didactic and the spatial organization must be unified for the correct integral development of the students.
\end{abstract}

Keywords: Higher education; spatial organization; classroom; evaluation

\title{
Resumo
}

Este estudo reflete a necessidade premente da qualidade e inovação da prática educativa na formação de estudantes, onde a variável organizacional de toda ação pedagógica é considerada um processo complexo que suporta a consideração de inúmeros elementos: recursos, momentos e espaços em que se desenvolvem os processos de ensinoaprendizagem. O objetivo da pesquisa é avaliar a organização espacial de uma sala de aula a partir da perspetiva de um grupo de estudantes universitários. Para isso, durante o ano letivo 2017/2018, através de uma metodologia de corte qualitativo, é examinado o caso numa sala de aula do ensino universitária. Foi usada uma amostra de 35 estudantes. Para isso, várias técnicas de pesquisa foram utilizadas: entrevistas semiestruturadas e observação participante. Os resultados indicam que, em geral, a organização espacial do centro e, em particular, da sala de aula, exerce um papel ativo em todo o processo de ensino-aprendizagem. Neste sentido, a didática e a organização espacial devem unir-se para o desenvolvimento correto e integral dos alunos.

Palavras-chave: Educação superior; organização espacial; sala de aula; avaliação 


\section{Introducción}

Actualmente, se sigue pensando que la calidad educativa depende exclusivamente del diseño curricular, de su puesta en práctica, de los/as profesionales que lo desarrollan y de los recursos que se usan para alcanzar sus objetivos (Romo, 2012). Sin embargo, se descuidan los aspectos contextuales, refiriéndonos a los factores estéticos condicionantes de la calidad educativa, el espacio escolar.

Hablamos, por tanto, del espacio escolar y, más concretamente, de ambiente de aprendizaje formado por aspectos físicos, metodológicos y sociales que configuran el espacio en el que las personas vivencian distintas experiencias (Romo, 2012). Asimismo, tras la revisión de la literatura, en cierto modo, podemos asegurar que el espacio es un agente educador esencial de toda planificación curricular, puesto que nos ayuda a definir la situación de enseñanza aprendizaje y nos permite crear un ambiente estimulante capaz de favorecer, por ejemplo, la autonomía y motivación de todas las personas que lo albergan (Bondioli y Nigito, 2011; Castro y Morales, 2015; Prakash, 2016).

Justamente, el objeto de este estudio se centra en evaluar la organización espacial de un aula desde la perspectiva de un grupo de estudiantes universitarios/as, ya que, siguiendo a Pozo (2017), el espacio del aula no debe ser algo superfluo y meramente decorativo, sino que debe ser una variable educativa que facilite la consecución de objetivos en relación a la puesta en marcha de los procesos de enseñanza aprendizaje.

\section{Método}

Se trata de un estudio de caso (Stake, 1995) en el que han participado 35 estudiantes (20 mujeres y 15 hombres) de una universidad española, con edades comprendidas entre los 21 y 29 años. Se han empleado entrevistas semiestructuradas y observación participante. Referente al análisis de los datos, se ha partido de un sistema provisional de categorías previamente identificadas (Strauss y Corbin, 1990). Una vez obtenidos y analizados los datos, se han retratado los temas emergentes que sintetizan el conjunto de la información obtenida mediante un análisis cualitativo del contenido del caso (Ying, 1993). De este modo, y mediante la triangulación, se ha tratado de reforzar la validez del estudio y aportar, en la medida de lo posible, respuesta al problema de investigación expuesto (Moral, 2006). 


\section{Resultados y discusión}

El sistema de categorías que ha emergido del presente estudio versa sobre cinco dimensiones clave: contexto, necesidades, obstáculos/limitaciones, puntos fuertes y propuesta de mejora (consúltese figura 1).

\begin{tabular}{|c|c|}
\hline DIMENSIONES & CUESTIONES \\
\hline \multirow{3}{*}{ CONTEXTO } & ¿Cómo definiría el espacio en el ámbito educativo? \\
\hline & $\begin{array}{l}\text { ¿En qué sentido cree que es importante el espacio en el proceso de } \\
\text { enseñanza aprendizaje? }\end{array}$ \\
\hline & ¿Qué papel juega el espacio en tu proceso de aprendizaje? \\
\hline \multirow{3}{*}{ NECESIDADES } & $\begin{array}{l}\text { ¿Cómo se ha sentido al entrar en el aula donde se ha impartido la } \\
\text { docencia? ¿Cómo te hubiera gustado sentirte? }\end{array}$ \\
\hline & ¿Qué necesidades cubre el aula? \\
\hline & ¿Cuáles no? \\
\hline \multirow{2}{*}{$\begin{array}{l}\text { OBSTÁCULOS/ } \\
\text { LIMITACIONES }\end{array}$} & ¿Cuáles son los principales obstáculos que presenta esta aula? \\
\hline & ¿Qué propondrías para mejorarlo? \\
\hline $\begin{array}{l}\text { PUNTOS } \\
\text { FUERTES }\end{array}$ & ¿Qué aspectos son los que más le gustan de nuestra aula? \\
\hline $\begin{array}{l}\text { PROPUESTA DE } \\
\text { MEJORA }\end{array}$ & ¿Cómo sería su aula ideal? ¿Por qué? \\
\hline
\end{tabular}

Figura 1.

Relación de categorías

- En cuanto al concepto del contexto (espacio) y necesidades, se puede observar como la mayoría de las personas entrevistadas consideran que éste es un lugar protagonista para el desarrollo de su proceso educativo, puesto que influye en las motivaciones, intereses y actitudes positivas hacia su permanencia en el aula. De igual modo, estos aspectos inciden en la necesidad de tomarlo como un factor didáctico, destacando su carácter polivalente, que faciliten diferentes dinámicas y estrategias como, por ejemplo, grupos de trabajos. Esto se corrobora en los trabajos de Iglesias (2008) y Prakash (2016), trabajos en los que se evidencia la necesidad de repensar el espacio en cuanto a su funcionalidad.

- Respecto a los obstáculos y limitaciones, se destacan las pequeñas dimensiones del aula, las cuales imposibilitan organizar el espacio de diferentes formas. En cuanto a los factores ambientales, se insiste en la orientación del aula y la incisión solar en la misma (son aspectos que dificultan en ciertos momentos, por ejemplo, la visibilidad), la sonoridad (en numerosas ocasiones, se acoplan sonidos provenientes 
de las aulas colindantes y no del pasillo central) y la temperatura (al no contar la clase con un termorregulador manual, en ocasiones, las condiciones ambientales afectan de diferentes maneras al bienestar común del alumnado, así como del resto de personas que participan en el aula). A este respecto, Bondioli y Nigito (2011) determinan la necesidad de atender a los efectos estéticos como condicionantes del ambiente generado en el espacio.

- En relación a los puntos fuertes, se destaca el acceso sin dificultad al aula, al encontrarse en la planta baja del edificio, facilitando la entrada a todas las personas. De igual modo, se observa la movilidad de los recursos básicos del aula, como el mobiliario movible, permitiendo la interacción social. Otro punto positivo es la presencia de recursos tecnológicos en la clase, como una mesa tecnológica y un proyector, recursos que permiten interactuar con las tecnologías de la información y la comunicación.

- Por último, se alude a las propuestas de mejora, refiriéndose al espacio funcional, concretamente, a la organización y al uso que se le da al mismo, y al espacio simbólico: colores del aula y decoración. Igualmente, se destaca la amplitud de la clase para la creación distintos espacios, tales como: biblioteca de aula, zona de confort y lectura, zona de trabajo individual con recursos móviles que permitan una mayor funcionalidad. Por su parte, se apunta hacia una mayor humanización y flexibilidad de la clase, principalmente para acondicionar la misma a los intereses de las personas que participan en ella. Finalmente, se ha destacado la presencia de plantas o mayor número de materiales básicos, tales como: estanterías, enchufes, papeleras, perchas, entre otros.

\section{Conclusiones}

Como conclusión final y teniendo en consideración toda la información expuesta a lo largo del presente documento, el espacio debe ser un elemento más de la planificación didáctica y, por tanto, es necesario estructurarlo y organizarlo adecuadamente. Así pues, tras haber culminado la investigación, se puede observar que la organización espacial del aula constituye un componente muy valioso para la puesta en marcha de los procesos de enseñanza aprendizaje, a lo que De Pablo y Trueba (1994, p. 14) añaden que "la organización del ambiente educativo estará en función de los protagonistas, de su edad, sus necesidades e intereses y de propiciar su encuentro y relación”. 
Por su parte, la propuesta de mejora que se puede incluir en este trabajo es el diseño de una clase alternativa a partir de los resultados obtenidos en esta evaluación, por ejemplo, utilizando el Design Thinking.

Finalmente, en cuanto a las limitaciones que hemos encontrado a lo largo de todo el proceso de investigación, hay que destacar las siguientes: variable temporal y escasa disposición por parte de algunos/as estudiante en el momento de ser entrevistados/as.

\section{Referencias}

Bondioli, A., y Nigito, G. (2011). Tiempos, espacios y grupos: el análisis y la evaluación de la organización en la escuela infantil: DAVOPSI. Barcelona: Graó.

Castro, M., y Morales, M. (2015). Los ambientes de aula que promueven el aprendizaje desde la perspectiva de los niños y niñas escolares. Revista Electrónica Educare, $19(3), 1-32$.

De Pablo, P., y Trueba, B. (1994). Espacios y recursos para ti, para mí, para todos. Diseño de ambientes en educación infantil. Madrid: Escuela Española.

Iglesias, L. (2008). Observación y evaluación del ambiente de aprendizaje en Educación Infantil. Dimensiones y variables a considerar. Revista Iberoamericana de Educación, 47, 49-70.

Moral, C. (2006). Criterios de validez en la investigación cualitativa actual. Revista de Investigación Cualitativa, 24(1), 147-164.

Pozo, M. (2017). La disolución del aula. Mapa de espacios arquitectónicos para un territorio pedagógico (Tesis Doctoral). Universidad de Sevilla, Andalucía.

Prakash, N. (2016). Diseños de espacios educativos. Rediseñar las escuelas para centrar el aprendizaje del alumno. Barcelona: SM.

Romo, V. (2012). Espacios educativos desafiantes en educación Infantil. En V. Peralta y L. Hernández (Coords.), Antología de experiencias de la educación inicial iberoamericana (pp. 141-145). Madrid: OEI y UNICEF.

Stake, R. (1995). The Art of Case Study Research. Thousand Oaks: Sage.

Strauss, A., y Corbin, J. (1990). Basics of Qualitative Research. Newbury Park: Sage.

Ying, R. (1993). Case study research design and methods applied. London: Sage. 